\title{
Sparing the hippocampus and the hypothalamic- pituitary region during whole brain radiotherapy: a volumetric modulated arc therapy planning study
}

P. Mehta ${ }^{1+}$, S. Janssen ${ }^{1,2^{*}+}$, F. B. Fahlbusch ${ }^{3}$, S. M. Schmid ${ }^{4,5}$, J. Gebauer ${ }^{4}$, F. Cremers' ${ }^{1}$, C. Ziemann ${ }^{1}$, M. Tartz ${ }^{2}$ and D. Rades ${ }^{1}$

\begin{abstract}
Background: Feasibility testing of a simultaneous sparing approach of hippocampus, hypothalamus and pituitary gland in patients undergoing whole-brain radiotherapy (WBRT) with and without a concomitant boost to metastatic sites.

Introduction: Cognitive impairment and hormonal dysfunction are common side effects of cranial radiotherapy. A reduced dose application to the patho-physiologically involved functional brain areas, i.e. hippocampus,

hypothalamus and pituitary gland, could reduce these common side effects. While hippocampal sparing is already a common practice to improve cognitive outcome, technical experience of additional combined sparing of the hypothalamus/pituitary gland (HT-P) is insufficient.

Methods: Twenty patients were included in the planning study. In 11 patients, a total dose of 36 Gy of WBRT (2 Gy per fraction) plus a simultaneous integrated boost (SIB) of 9 Gy (0.5 Gy per fraction, total dose: 45 Gy) to the brain metastases was applied. In 9 patients, prophylactic cranial irradiation (PCI) was simulated with a total dose of $30 \mathrm{~Gy}$ (2 Gy per fraction). In both patient cohorts, a sparing approach of the hippocampus and the HT-P area was simulated during WBRT. For all treatment plans, volumetric modulated arc therapy (VMAT) was used. Quality assurance included assessment of homogeneity, conformality and target coverage.

Results: The mean dose to the hippocampus and HT-P region was limited to less than $50 \%$ of the prescribed dose to the planning target volume (PTV) in all treatment plans. Dose homogeneity (HI) of the target volume was satisfying (median $\mathrm{HI}=0.16$ for WBRT+SIB and 0.1 for $\mathrm{PCl}$ ) and target coverage (conformation number, CN) was not compromised (median $\mathrm{CN}=0.82$ for $\mathrm{SIB}$ and 0.86 for $\mathrm{PCI}$ ).

(Continued on next page)
\end{abstract}

\footnotetext{
* Correspondence: Stefan.janssen@UKSH.de

${ }^{\dagger} \mathrm{P}$. Mehta and S. Janssen contributed equally to this work.

'Department of Radiation Oncology, University of Lübeck, Lübeck, Germany

${ }^{2}$ Private Practice of Radiation Oncology, Hannover, Germany

Full list of author information is available at the end of the article
}

C C The Author(s). 2020 Open Access This article is licensed under a Creative Commons Attribution 4.0 International License, which permits use, sharing, adaptation, distribution and reproduction in any medium or format, as long as you give appropriate credit to the original author(s) and the source, provide a link to the Creative Commons licence, and indicate if changes were made. The images or other third party material in this article are included in the article's Creative Commons licence, unless indicated otherwise in a credit line to the material. If material is not included in the article's Creative Commons licence and your intended use is not permitted by statutory regulation or exceeds the permitted use, you will need to obtain permission directly from the copyright holder. To view a copy of this licence, visit http://creativecommons.org/licenses/by/4.0/ The Creative Commons Public Domain Dedication waiver (http://creativecommons.org/publicdomain/zero/1.0/) applies to the data made available in this article, unless otherwise stated in a credit line to the data. 
(Continued from previous page)

Conclusion: Simultaneous dose reduction to the hippocampus and the HT-P area did not compromise the PTV coverage in patients undergoing WBRT+SIB or PCI using VMAT. While the feasibility of the presented approach is promising, prospective neurologic, endocrine outcome and safety studies are required.

Keywords: Whole brain radiotherapy (WBRT), Brain metastases, Hippocampus sparing, Hypothalamus, Pituitary gland, Volumetric modulated arc therapy (VMAT)

\section{Background}

Up to $30 \%$ of cancer patients develop brain metastases during their disease [1]. Despite the increasing use of high precision radiation techniques for small volumes [2-4], whole brain radiotherapy (WBRT) remains the treatment of choice for patients with multiple brain metastases [5] as well as for prophylactic cranial irradiation (PCI) in patients with small cell lung cancer (SCLC) [6, 7]. However, cognitive and neuroendocrine impairment following cranial radiotherapy remains a concern. Mechanistically, damage to the stem cells within the hippocampus might play a major role in the observed memory decline [8]. In line with this finding, Gondi et al. were able to show that conformal avoidance of the hippocampus during WBRT was associated with preservation of memory function and quality of life (QoL), as compared to a non-sparing historical series [9]. Apart from neurocognitive decline, another common sequela of cranial radiation therapy is functional endocrine impairment due to critical doses to the hypothalamus and the pituitary gland. A significant percentage of patients with brain tumors [10-12] and head and neck cancer [13-15] develop hormonal deficiencies after radiotherapy. At the time of our research period, endocrine follow-up data after WBRT was scarce. Nevertheless, it has been shown that hormonal changes can occur after applied doses as low as $18 \mathrm{~Gy}$ in patients with radiotherapy to head and neck cancers and brain tumors [13]. This dichotomy led us to investigate a combined sparing approach involving both the hippocampal and the hypothalamus/pituitary gland (HT-P) area during WBRT. In our planning study we examined the feasibility of such an approach using volumetric modulated arc therapy (VMAT).

\section{Methods}

The computed tomography (CT)-data sets of 20 patients who previously received WBRT in our institution from 2017 to 2019 were included. The CT-scans were performed with a Siemens Biograph $40 \mathrm{~m}$ with a slice thickness of 3 $\mathrm{mm}$. To facilitate contouring of the brain structures and metastases T1-weighted contrast-enhanced magnetic resonance images (MRI) were fused to the planning CT. In addition to the contoured hippocampus (according the RTOG 0933 study [9]), the hypothalamus and pituitary gland (including the pituitary stalk) were contoured and planning risk volumes (PRV) were created using a 5-mm margin $[16,17]$. CT data sets with metastases within $5 \mathrm{~mm}$ around the avoidance structures were excluded from this planning study. Further, the whole brain planning target volume (PTV) was contoured and cropped by the hippocampus and HT-P as a planning risk volume (PRV). An auxiliary PTV structure consisting of the part of the optimization PTV surrounding the HT-P and hippocampus helped to control the dose drop in the immediate vicinity of the hippocampus and the HT-P (Fig. 1).

Treatment plans were then created using Eclipse 15.5 (Varian Medical Systems, Inc., Palo Alto, CA, USA) for a Clinac DHX linear accelerator equipped with a Millennium 120 MLC. The Photon Optimizer (PO) and Anisotropic Analytical Algorithm (AAA), both in versions 15.5.11, were utilized [18]. The normalization point was set to $100 \%$ of the mean dose of the target volume. All treatment plans were created on the basis of sparing both the hippocampal and the HT-P area, concomitantly avoiding dose peaks to lenses, eyes, chiasm, optical nerves and brainstem. 11 of 20 patients were planned with a dose of $18 \times 2 \mathrm{~Gy}=36 \mathrm{~Gy}$ as a WBRT with a SIB $(18 \times 0.5 \mathrm{~Gy}=9$ Gy, total dose: $45 \mathrm{~Gy}$ ) to the metastases resulting in a total dose of $18 \times 2.5 \mathrm{~Gy}$ in the area of the SIB, whereas the other 9 patients were planned as a PCI with a dose of $15 \times 2$ Gy $=30$ Gy. A VMAT treatment plan was generated individually for each patient by a medical physicist (P.M.). Table 1 summarizes patient and treatment parameters.

Two different planning approaches were used for therapeutic WBRT with SIB to metastases and for PCI. Both were planned with three 6 Megavolt (MV) photon beam full arcs using the VMAT technique. For the treatment plans including SIB two of the arcs had a rotation collimator of $320^{\circ}$ and $40^{\circ}$, while in the third arc the collimator was rotated by $90^{\circ}$ and the jaws were adjusted to the length of the organs of risk (OAR), i.e. the hypothalamus, hippocampus and pituitary gland to guarantee homogenous dose coverage between the OAR. For the PCI plans the collimator angles were $280^{\circ}$, $90^{\circ}$ and $11^{\circ}$ respectively. Couch rotations of $15^{\circ}, 0^{\circ}$, and $345^{\circ}$ were used. These different techniques provided the optimal combined sparing approaches of both hippocampal/HT-P structures and ocular lenses, while concomitantly ensuring the best PTV-dose coverage.

The treatment plans were generated with the goal to achieve a dose of lower than $50 \%$ of the prescription dose 


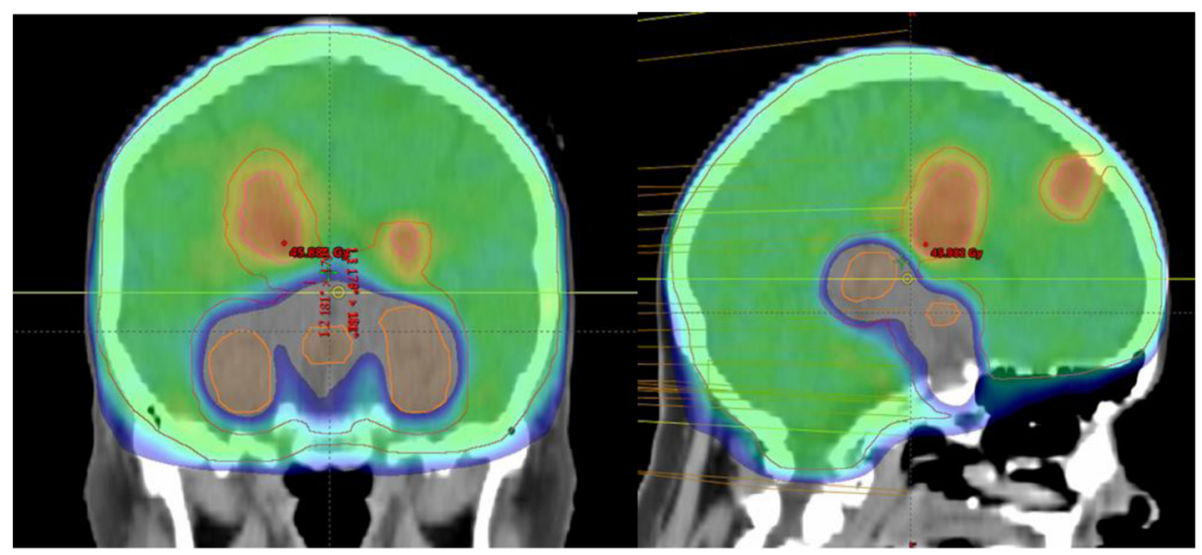

Fig. 1 a and b Representative example of a VMAT plan with avoidance of the hippocampus and HT-P area and SIB (color-wash, coronar (a) and sagittal (b) view, dose levels: blue: 25-32 Gy, cyan: 32-37 Gy, green: 37-42 Gy, yellow: 42-44 Gy, orange: 44-46 Gy, red: > 46 Gy)

of the PTV in the sparing regions without compromising conformal dose coverage. Additionally, mean maximum doses to the ocular lenses were kept below 10 Gy. Homogeneity index (HI) was calculated as follows [19]:

$$
\mathrm{HI}=\frac{\mathrm{D}_{2 \%}-\mathrm{D}_{98 \%}}{\mathrm{D}_{\text {median }}}
$$

Where $\mathrm{D}_{\mathrm{x} \%}$ is the dose which is at least delivered to $\mathrm{x} \%$ of the volume and $\mathrm{D}_{\text {median }}$ is the median dose. Smaller values of HI correspond to a more homogeneous irradiation of the target volume, and a value of 0 corresponds to a completely homogeneous dose distribution within the target [16].

Conformality index or conformation number $(\mathrm{CN})$ was calculated according the formula introduced by van't Riet et al. in 1997 [20]:

Table 1 Patient and treatment related parameters, $\mathrm{HI}=$ homogeneity index, $\mathrm{HT}-\mathrm{P}=$ hypothalamus-pituitary area, $\mathrm{CN}=\mathrm{conformation}$ number, SIB: simultaneous integrated boost, n.a. =not applicable; $m=$ male, $f=$ female

\begin{tabular}{|c|c|c|c|c|c|c|c|c|c|c|}
\hline & $\begin{array}{l}\text { PTV volume } \\
{\left[\mathrm{cm}^{3}\right]}\end{array}$ & $\begin{array}{l}\text { Hippocampal } \\
\text { volume }\left[\mathrm{cm}^{3}\right]\end{array}$ & $\begin{array}{l}\text { Hippocampal HT-P- }+5 \mathrm{~mm} \\
\text { volumes } V_{\text {PRV }}\left[\mathrm{cm}^{3}\right]\end{array}$ & $\begin{array}{l}\text { Number of } \\
\text { metastases }\end{array}$ & $\begin{array}{l}\text { Metastases } \\
\text { volumes }\left[\mathrm{cm}^{3}\right]\end{array}$ & $\begin{array}{l}\% \text { SIB of } \\
\text { PTV }\end{array}$ & $\begin{array}{l}\text { D95 } \\
(\%)\end{array}$ & $\begin{array}{l}\text { Hippocampal HT-P-+ } 5 \text { mm } \\
\text { mean dose DPRV }[G y]\end{array}$ & $\mathrm{HI}$ & $C N$ \\
\hline 1 & 1748.1 & 12.6 & 58.9 & n.a. & n.a. & & 96.4 & 12.8 & 0.09 & 0.85 \\
\hline 2 & 2048.5 & 12.6 & 58.0 & n.a. & n.a. & & 95.9 & 14.1 & 0.17 & 0.86 \\
\hline 3 & 2140.7 & 6.1 & 43.9 & n.a. & n.a. & & 95.5 & 14.6 & 0.12 & 0.86 \\
\hline 4 & 2083.5 & 10.7 & 65.6 & n.a. & n.a. & & 96.4 & 14.2 & 0.09 & 0.86 \\
\hline 5 & 1974.4 & 4.5 & 43.2 & n.a. & n.a. & & 95.3 & 14.9 & 0.12 & 0.86 \\
\hline 6 & 1797.2 & 4.7 & 59.6 & n.a. & n.a. & & 95.3 & 14.9 & 0.11 & 0.84 \\
\hline 7 & 1805.3 & 8.1 & 51.4 & n.a. & n.a. & & 96.7 & 15 & 0.08 & 0.86 \\
\hline 8 & 1521.4 & 10.4 & 58.8 & n.a. & n.a. & & 96.4 & 14.9 & 0.09 & 0.82 \\
\hline 9 & 1991.4 & 6.7 & 45.9 & n.a. & n.a. & & 96.4 & 15 & 0.09 & 0.87 \\
\hline 10 & 1593.9 & 7.4 & 47.9 & 2 & 5.2 & 0.33 & 98.1 & 15.1 & 0.11 & 0.84 \\
\hline 11 & 1924.7 & 22.4 & 51.2 & 1 & 9.4 & 0.49 & 98.1 & 15 & 0.13 & 0.85 \\
\hline 12 & 1761.8 & 2.7 & 38.9 & 2 & 7.1 & 0.40 & 98.0 & 14.1 & 0.14 & 0.84 \\
\hline 13 & 1436.4 & 4.0 & 39.6 & 1 & 15.9 & 1.11 & 97.8 & 14.9 & 0.19 & 0.81 \\
\hline 14 & 1860.0 & 4.7 & 44.1 & 1 & 4.1 & 0.22 & 98.0 & 13.2 & 0.16 & 0.86 \\
\hline 15 & 1802.7 & 6.0 & 42.2 & 9 & 31.7 & 1.76 & 97.6 & 14 & 0.214 & 0.79 \\
\hline 16 & 2286.7 & 10.1 & 65.8 & 3 & 73.0 & 3.19 & 97.3 & 14.2 & 0.16 & 0.79 \\
\hline 17 & 1641.1 & 8.8 & 53.6 & 1 & 6.5 & 0.40 & 98.3 & 13.6 & 0.17 & 0.85 \\
\hline 18 & 2029.0 & 9.6 & 59.4 & 1 & 105.0 & 5.17 & 97.5 & 15.4 & 0.15 & 0.72 \\
\hline 19 & 1941.3 & 2.5 & 34.3 & 4 & 20.6 & 1.06 & 97.6 & 14.8 & 0.23 & 0.82 \\
\hline 20 & 1781.4 & 5.8 & 41.5 & 2 & 19.8 & 1.11 & 97.6 & 15 & 0.13 & 0.82 \\
\hline
\end{tabular}




$$
\mathrm{CN}=\frac{\mathrm{TV}_{\mathrm{RI}}}{\mathrm{TV}} \times \frac{\mathrm{TV}_{\mathrm{RI}}}{\mathrm{V}_{\mathrm{RI}}}
$$

Where $T V_{\mathrm{RI}}$ is target volume covered by the reference isodose (95\% isodose), TV is the target volume and $\mathrm{V}_{\mathrm{RI}}$ is the volume of the reference isodose ( $95 \%$ isodose). The conformation number reaches a value between 0 and 1 . A value of 1 represents a reference isodose covering the exact target volume without irradiation of healthy tissue and indicates optimal conformation. On the other hand, a value of 0 equals no conformation at all [20].

The target coverage (TC) was measured as the volume within the target receiving a dose greater or equal to the prescription dose (VTpres) divided by the target volume (TV) [21].

$$
\mathrm{TC}=\frac{\mathrm{V}_{\mathrm{Tpres}}}{\mathrm{TV}}
$$

No patients consent was obtained as all patients' data were irreversibly anonymized before analysis. The in silico analysis included CT database data only. In this form, the study was approved by the local ethics committee of the University of Lübeck, Germany (reference number: 19-075A).

\section{Results}

The median total brain volume including the avoidance region was $1832.7 \mathrm{~cm}^{3}$ (range: $1436.4 \mathrm{~cm}^{3}-2286.7 \mathrm{~cm}^{3}$ ). The median volume of the hippocampus/HT-P area (including a margin of $5 \mathrm{~mm}$ ) was $43.9 \mathrm{~cm}^{3}$ (range: $34.3-$ $65.8 \mathrm{~cm}^{3}$ ). For patients receiving a SIB to brain metastases, the median value of the SIB volume was $15.9 \mathrm{~cm}^{3}$ (range: $5.2-105.0 \mathrm{~cm}^{3}$ ). Number of metastases treated with a SIB ranged from 1 to 9 (median: 2). The median percentage of the SIB volumes of the entire planning treatment volume (PTV) was 3.3\% (range: 1.3-11.6\%). In the 11 WBRT plans including SIB to brain metastases the median delivered dose to the hippocampus/HT-P area was 14.9 Gy (range: $13.2-16.2$ Gy). In the 9 PCI plans the delivered dose to the hippocampus/HT-P could be held below 15Gy (median: $14.8 \mathrm{~Gy}$, range: 12.8-15.0 Gy). Maximum dose to the ocular lenses was limited to $10 \mathrm{~Gy}$ for each patient. Median maximum dose for all plans within in lenses was 8.3 Gy. The corresponding values for the eyes, the brainstem, chiasma and optic nerves were 25/30 Gy, 31/40 Gy, 29/33 Gy and 31/ $39 \mathrm{~Gy}$ for prophylactic and therapeutic plans, respectively. The median homogeneity index was 0.16 (range: 0.11-0.23) for the SIB plans and 0.10 (range: 0.08-0.17) for the PCI plans. The median $\mathrm{D}_{95 \%}$ for the WBRT plans including SIB 97.8\% (range: 97.3-98.3\%) and 96.4\% (range: 95.5-96.7\%) for PCI plans. The median conformity index was 0.85 for all plans, 0.82 for the therapeutic plans including SIB (range: 0.72-0.86) and 0.86 for the PCI plans (range: $0.82-0.87$ ). The target coverage was 0.7 (range: 6.3-8.7) for prophylactic and therapeutic plans, respectively. Figure $2 \mathrm{a}$ and $\mathrm{b}$ show the dose volume histograms (DVH) for SIB plans and PCI plans.

\section{Discussion}

WBRT for brain metastases can impair neuro-cognitive functions in terms of memory loss and reduced QoL [8]. Neural stem cells within the hippocampus may play an important role in this patho-mechanism. In RTOG 0933, avoidance of the hippocampus during WBRT was associated with preservation of memory and QoL as compared with a non-sparing historical series [9]. Preliminary analysis of a randomized phase III trial confirms the hypothesis of preserved neurocognitive function while achieving similar intracranial control and survival [22].

Functional endocrine deficiencies after brain radiotherapy are common [23]. Long term follow-up studies indicate that radiation induced HT-P dysfunction may occur in up to $80 \%$ of patients and is often associated with an adverse impact on growth, body image, skeletal health, fertility, sexual function and physical and psychological health [24]. Several studies showed the hormonal impairment to be dose-dependent with an increased incidence at doses above 30 Gy [17]. Until now, most data of radiation induced endocrine sequelae in adults originate from patients being treated for head and neck cancer and non-pituitary brain tumors. Endocrine follow-up data on hormonal changes after WBRT are scarce [23]. As the hormonal impairment is described to be dosedependent, limiting the dose to the HT-P area could be beneficial. During WBRT, this could be realized with a sparing approach analogue to the hippocampus sparing technique introduced by Gondi et al. [9], as previously discussed by us [23]. Arguments against a theoretical benefit of such a sparing approach are the limited life expectancy of patients with brain metastases and lower doses to the HT-P region compared to RT in head and neck cancers and brain tumors. Still, in a current review of literature we could reveal a potential effect of RT for doses of less than 30 Gy being within the dose range of WBRT [23]. Moreover, the potential negative endocrine effect might already occur as early as within the first year after RT [23]. This is of relevance especially for patients with a more favorable prognosis, e.g. for patients with good performance status and a limited tumor burden or in the prophylactic setting in SCLC patients. For this reason, we carried out a planning approach for both, therapeutic and prophylactic scenarios encompassing a combined sparing of the hippocampus and the HT-P area.

According to the present VMAT planning study, simultaneous sparing the hippocampus and the HT-P axis 


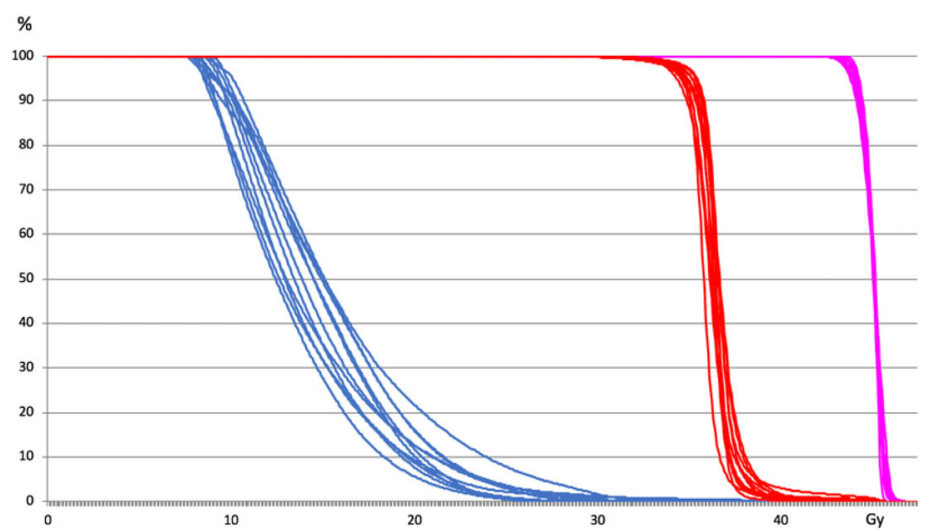

a

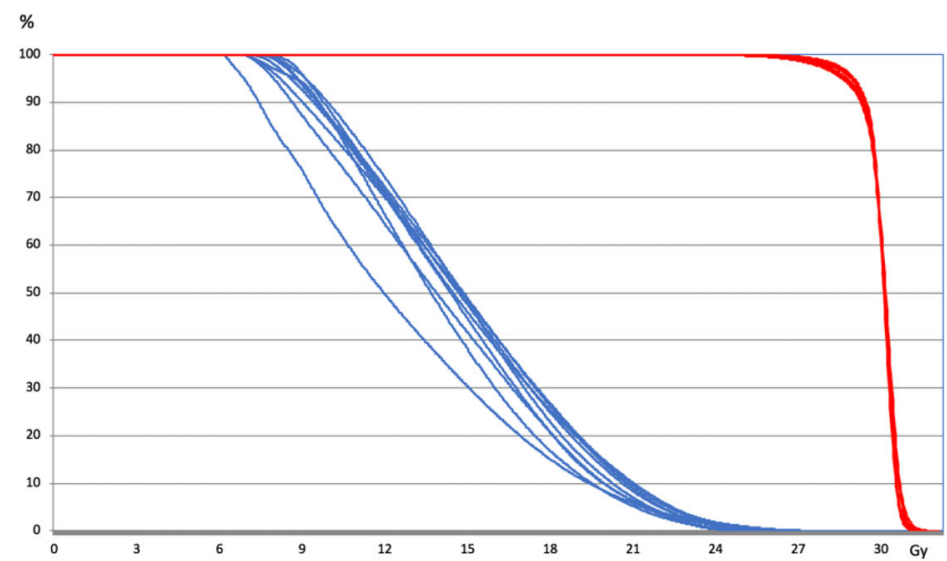

$\mathrm{b}$

Fig. 2 a and b Representative cumulative dose volume histograms (DVH) for SIB plans (a) and PCI plans (b). Blue: sparing region, red: PTV whole brain, pink: SIB

was feasible. The dose to the avoidance regions could be limited to less than $50 \%$ of the prescribed doses to the PTV. For the hippocampus, several dose constraints were suggested in previous studies. In the RTOG 0933 protocol, the dose to $100 \%$ of hippocampus did not exceed 9 Gy $\left(D_{100 \%}<9 \mathrm{~Gy}\right)$, and the maximal hippocampal dose did not exceed 16 Gy [9]. Other studies involving hippocampal sparing approaches in patients treated with WBRT delivered mean doses to the hippocampi ranging from 5 Gy to $20 \mathrm{~Gy}$, depending on radiation techniques and total doses [24]. Until now, no threshold dose for the HT-P area has been established. Kyriakakis et al. assessed the effects of cranial RT on pituitary function in adults with gliomas distant to the HT-P axis. The dose exposure of the HT-P axis was correlated with individual axis dysfunction to establish dose thresholds. The authors argued for the implementation of long-term endocrine surveillance in RT cases exceeding $30 \mathrm{~Gy}$ to the HT-P axis [25].
In a study by Fan et al., in which the hippocampus and the HT-P area were spared simultaneously using intensity modulated radiotherapy (IMRT), the hippocampus received a mean dose of $9.6 \mathrm{~Gy}$, and the hypothalamus and the pituitary gland mean doses of 11.06 and 10.66 Gy, respectively [16]. In the present study, the mean doses to the hippocampus and the HT-P area were 15 Gy i.e. comparably higher. This finding might result from higher doses to the total brain volume in our study when compared to previous studies (36 Gy and $30 \mathrm{~Gy}$ versus $30 \mathrm{~Gy}$ and $25 \mathrm{~Gy}$ ) $[9,16]$. In the present study, the metastases even received $45 \mathrm{~Gy}$. Moreover, we also attempted to spare the ocular lenses during WBRT and to achieve conformal dose coverage. However, our boost doses to a maximum 45Gy (normo-fractionated) are a rather cautious approach and are currently under discussion.

In contrast to Fan et al., who were the first group describing a combined sparing approach of the 
hippocampus and the HT-P area using IMRT, we chose a VMAT approach. For hippocampal sparing during WBRT (without the HT-P area), the use of VMAT was shown to significantly improve dose distribution in terms of target coverage and homogeneity [26-28]. In the study of Sood et al., the use of a VMAT-technique also reduced mean and maximum doses to other organs at risk (OAR) such as cochleae and parotid glands [29]. These promising results inspired us to use VMAT in our approach to spare the hippocampus and the HT-P. In addition, when comparing our VMAT data to the IMRT approach used by Fan et al, we were able to achieve less heterogeneity with respect to the dose coverage of the PTV (homogeneity index: 0.23 vs. 0.10 and 0.16 in our study). Further, we kept the maximum dose to the ocular lenses below $10 \mathrm{~Gy}$; no information concerning the doses to the lenses was provided by Fan et al. [16]. Another advantage of VMAT is its faster treatment delivery. For hippocampal sparing in WBRT, Wang et al. demonstrated a significant shorter treatment time of approximately $25 \%$ using VMAT in comparison to IMRT [30]. Moreover, Rong et al. found faster treatment delivery of VMAT when compared to IMRT [31].

For hippocampal sparing in WBRT, slightly superior homogeneity indices and target coverage were found for tomotherapy when compared to IMRT and VMAT [31, 32]. However, the availability of tomotherapy is limited, and the treatment planning time is significantly longer. Another possibility to improve the quality of the treatment planning could be an inclined head position [33, 34]. In a recently published study, Zheng et al. showed feasibility using VMAT and tomotherapy for HT-P and hippocampal axis sparing for cranio-spinal irradiation. They also found that VMAT was able to achieve good conformality [35].

In line with data from hippocampal sparing WBRT, simultaneous sparing of the hippocampus and HT-P via VMAT delivered highly conformal and fast-to-apply treatment plans, resulting in a direct advantage for patients in their daily treatment sessions.

A sparing approach of certain brain regions bears the risk of jeopardizing oncologic outcomes in terms of intracranial control and consecutive overall survival. Therefore, the estimated risk of metastases within spared structures and their proximity have to be taken into careful consideration. Gondi et al. deemed hippocampus sparing WBRT safe with an estimated risk of peri-hippocampal metastases of $8.6 \%$ [36]. Our group has recently analyzed 865 patients with 4280 metastases and showed an incidence of involvement of the HT-P area of approximately 4\% [37]. Against that background, an approach of sparing the HT$\mathrm{P}$ area in addition to the hippocampus during WBRT appears reasonable. Thus, in order to reveal a clinical meaningful effect of HT-P region sparing within WBRT, a prospective study is planned evaluating a sparing approach with simultaneous avoidance of the hippocampus and the HT-P area including endocrine follow-up. The current planning study, which is a prerequisite for the planned prospective trial, showed technical feasibility of such an approach using VMAT even for dose escalation with a SIB. In the absence of safety data, the presented approach remains experimental and should not be applied outside a clinical study.

\section{Conclusion}

Simultaneous dose reduction to the hippocampus and the HT-P area did not compromise the PTV coverage in patients undergoing WBRT+SIB or PCI when using VMAT. While the feasibility of the presented approach is promising, prospective neurologic and endocrine outcome studies are required to properly evaluate the usefulness of such an approach.

\section{Abbreviations \\ CN: Conformation number; DVH: Dose volume histograms; IMRT: Intensity modulated radiotherapy; HI: Homogeneity index; HT-P: Hypothalamus/ pituitary gland; PCl: Prophylactic cranial irradiation; PTV: Planning target volume; SCLC: Small cell lung cancer; SIB: Simultaneous integrated boost; TC: Target coverage; OAR: Organs at risk; VMAT: Volumetric modulated arc therapy; WBRT: Whole brain radiotherapy}

\section{Acknowledgements}

Not applicable.

\section{Authors' contributions}

All authors have read and approved the final version of the manuscript. Idea and conception: SJ, DR, FF, PM, FC, MT, SS. Planning; PM, MT, FC, CZ.

Interpretation: SJ, DR, FF, FC, MT, CZ SS, JG. Manuscript writing: SJ, FF, JG, SS, DR.

\section{Funding}

None.

\section{Availability of data and materials}

The datasets used and/or analyzed during the current study are available from the corresponding author on reasonable request.

\section{Ethics approval and consent to participate}

Administrative permission was acquired to access data used in the research. The analysis was not an intervention study. Only fully anonymized CT dataset were used. The study was approved by the ethics committee of the University of Lübeck with administrative permission to access the raw data (reference number: 19-075A). The study was conducted in accordance with the principles laid out in the Declaration of Helsinki and in accordance with the principles of Good Clinical Practice.

\section{Consent for publication}

Not applicable.

\section{Competing interests}

DR and SJ are editorial board members of BMC Cancer, all authors declare that they have no competing interests.

\section{Author details}

${ }^{1}$ Department of Radiation Oncology, University of Lübeck, Lübeck, Germany. ${ }^{2}$ Private Practice of Radiation Oncology, Hannover, Germany. ${ }^{3}$ Department of Pediatrics and Adolescent Medicine, Friedrich-Alexander-University of Erlangen-Nürnberg, Erlangen, Germany. ${ }^{4}$ Institute for Endocrinology and Diabetes, University of Lübeck, Lübeck, Germany. ${ }^{5}$ German Center for Diabetes Research (DZD), Neuherberg, Germany. 
Received: 23 March 2020 Accepted: 18 June 2020

Published online: 30 June 2020

\section{References}

1. Khuntia D, Brown P, Li J, Mehta MP. Whole-brain radiotherapy in the management of brain metastasis. J Clin Oncol. 2006;24(8):1295-304.

2. Steinmann D, Vordermark D, Gerstenberg W, Aschoff R, Gharbi N, Müller A, Schäfer C, Theodorou M, Wypior HJ, Geinitz H. Quality of life working Group of the German Radiation Oncology Society (DEGRO). Quality of life in patients with limited (1-3) brain metastases undergoing stereotactic or whole brain radiotherapy: a prospective study of the DEGRO QoL working group. Strahlenther Onkol. 2020;196(1):48-57.

3. Gregucci F, Fiorentino A, Corradini S, Figlia V, Mazzola R, Ricchetti F, Ruggieri R, Alongi F. Linac-based radiosurgery or fractionated stereotactic radiotherapy with flattening filter-free volumetric modulated arc therapy in elderly patients : a mono-institutional experience on 110 brain metastases. Strahlenther Onkol. 2019;195(3):218-25.

4. Ishihara T, Yamada K, Harada A, Isogai K, Tonosaki Y, Demizu Y, Miyawaki D, Yoshida K, Ejima Y, Sasaki R. Hypofractionated stereotactic radiotherapy for brain metastases from lung cancer : evaluation of indications and predictors of local control. Strahlenther Onkol. 2016;192(6):386-93.

5. Oehlke O, Wucherpfennig D, Fels F, Frings L, Egger K, Weyerbrock A, Prokic V, Nieder C, Grosu AL. Whole brain irradiation with hippocampal sparing and dose escalation on multiple brain metastases: local tumour control and survival. Strahlenther Onkol. 2015;191(6):461-9.

6. Aupérin A, Arriagada R, Pignon JP, Le Péchoux C, Gregor A, Stephens RJ, Kristjansen PE, Johnson BE, Ueoka H, Wagner H, Aisner J. Prophylactic cranial irradiation for patients with small-cell lung cancer in complete remission. Prophylactic cranial irradiation overview collaborative group. $\mathrm{N}$ Engl J Med. 1999;341(7):476-84.

7. Slotman BJ, van Tinteren H, Praag JO, Knegjens JL, El Sharouni SY, Hatton M, Keijser A, Faivre-Finn C, Senan S. Use of thoracic radiotherapy for extensive stage small-cell lung cancer: a phase 3 randomised controlled trial. Lancet. 2015;385(9962):36-42.

8. Gondi V, Tomé WA, Mehta MP. Why avoid the hippocampus? A comprehensive review. Radiother Oncol. 2010;97(3):370-6.

9. Gondi V, Pugh SL, Tome WA, Caine C, Corn B, Kanner A, et al. Preservation of memory with conformal avoidance of the hippocampal neural stemcell compartment during whole-brain radiotherapy for brain metastases (RTOG 0933): a phase II multi-institutional trial. J Clin Oncol. 2014;32(34): 3810-6.

10. Kyriakakis N, Lynch J, Orme SM, Gerrard G, Hatfield P, Loughrey C, Short SC, Murray RD. Pituitary dysfunction following cranial radiotherapy for adultonset nonpituitary brain tumours. Clin Endocrinol. 2016;84(3):372-9.

11. Handisurya A, Rumpold T, Caucig-Lütgendorf C, Flechl B, Preusser M, IlhanMutlu A, Dieckmann K, Widhalm G, Grisold A, Wöhrer A, Hainfellner J, Ristl R, Kurz C, Marosi C, Gessl A, Hassler M. Are hypothyroidism and hypogonadism clinically relevant in patients with malignant gliomas? A longitudinal trial in patients with glioma. Radiother Oncol. 2019;130:139-48.

12. Madaschi S, Fiorino C, Losa M, Lanzi R, Mazza E, Motta M, Perna L, Brioschi E, Scavini M, Reni M. Time course of hypothalamic-pituitary deficiency in adults receiving cranial radiotherapy for primary extrasellar brain tumors. Radiother Oncol. 2011;99(1):23-8.

13. Appelman-Dijkstra NM, Malgo F, Neelis KJ, Coremans I, Biermasz NR, Pereira AM. Pituitary dysfunction in adult patients after cranial irradiation for head and nasopharyngeal tumours. Radiother Oncol. 2014;113(1):102-7.

14. Seland M, Bjøro T, Furre T, Schreiner T, Bollerslev J, Fosså SD, et al. Hormonal dysfunction is frequent in cancer survivors treated with radiotherapy to the head and neck region. J Cancer Surviv. 2015;9(4):630-40.

15. Ipekci SH, Cakir M, Kiyici A, Koc O, Artac M. Radiotherapy-induced hypopituitarism in nasopharyngeal carcinoma: the tip of an iceberg. Exp Clin Endocrinol Diabetes. 2015;123(7):411-8.

16. Fan XW, Wang JQ, Wu JL, Wang HB, Wu KL. Simultaneously avoiding the hippocampus and hypothalamic-pituitary axis during whole brain radiotherapy: A planning study. Med Dosim. 2019:44(2):130-5.

17. Scoccianti S, Detti B, Gadda D, Greto D, Furfaro I, Meacci F, Simontacchi G, Di Brina L, Bonomo P, Giacomelli I, Meattini I, Mangoni M, Cappelli S, Cassani S, Talamonti C, Bordi L, Livi L. Organs at risk in the brain and their dose-constraints in adults and in children: a radiation oncologist's guide for delineation in everyday practice. Radiother Oncol. 2015;114(2):230-8.
18. Ulmer W, Harder D. A triple Gaussian pencil beam model for photon beam treatment planning. Z Med Phys. 1995;5:25-30.

19. International Commission on Radiation Units and Measurements. Prescribing, Recording, and Reporting Photon-Beam Intensity-Modulated Radiation Therapy (IMRT): ICRU Report 83. 2010.

20. van't Riet A, Mak AC, Moerland MA, Elders LH, van der Zee W. A conformation number to quantify the degree of conformaliy in brachytherapy and external beamirradiation: application to the prostate. Int J Radiat Oncol Biol Phys. 1997;37(3):731-6.

21. Levra NG, Sicignano G, Fiorentino A, Fersino S, Ricchetti F, Mazzola R, Naccarato S, Ruggieri R, Alongi F. Whole brain radiotherapy with hippocampal avoidance and simultaneous integrated boost for brain metastases: a Dosimetric volumetric-modulated arc therapy study. Radiol Med. 2016;121(1):60-9.

22. Gondi V, Deshmukh S, Brown PD, Wefel JS, Tome WA, Bruner DW, Bovi JA, Robinson CG, Khuntia D, Grosshans DR, Konski AA, Roberge D, Kundapur V, Devisetty K, Shah SA, Usuki KY, Anderson BM, Metha MP, Kachnic LA. Preservation of neurocognitive function (NCF) with conformal avoidance of the Hippocampus during whole- brain radiotherapy (HA-WBRT) for brain metastases: preliminary results of phase III trial NRG oncology CC001. Int J Radiat Oncol Biol Phys. 1607;102(5).

23. Mehta P, Fahlbusch FB, Rades D, Schmid SM, Gebauer J, Janssen S. Are hypothalamic- pituitary (HP) axis deficiencies after whole brain radiotherapy (WBRT) of relevance for adult cancer patients? - a systematic review of the literature. BMC Cancer. 2019;19(1):1213.

24. Darzy KH. Radiation-induced hypopituitarism. Curr Opin Endocrinol Diabetes Obes. 2013;20(4):342-53.

25. Kyriakakis N, Lynch J, Orme SM, Gerrard G, Hatfield P, Short SC, Loughrey C, Murray RD. Hypothalamic-pituitary axis irradiation dose thresholds for the development of hypopituitarism in adult-onset gliomas. Clin Endocrinol (Oxf). 2019;91(1):131-40.

26. Zieminski S, Khandekar M, Wang Y. Assessment of multi-criteria optimization (MCO) for volumetric modulated arc therapy (VMAT) in hippocampal avoidance whole brain radiation therapy (HA-WBRT). Australas Phys Eng Sci Med. 2015;38(4):767-75.

27. Wang BH, Hua W, Gu X, Wang XL, Li J, Liu LQ, Huang YX. Dosimetric study of different radiotherapy planning approaches for hippocampal avoidance whole-brain radiation therapy (HA-WBRT) based on fused CT and MRI imaging. Med Dosim. 2016;41(1):15-21.

28. Lee K, Lenards $\mathrm{N}$, Holson J. Whole-brain hippocampal sparing radiation therapy: volume-modulated arc therapy vs intensity-modulated radiation therapy case study. PLoS One. 2015;10(4):e0126222.

29. Sood S, Pokhrel D, McClinton C, Lominska C, Badkul R, Jiang H, Wang F. Volumetric-modulated arc therapy (VMAT) for whole brain radiotherapy: not only for hippocampal sparing, but also for reduction of dose to organs at risk. J Appl Clin Med Phys. 2018;19(2):184-90.

30. Pokhrel D, Sood SS, Kumar P, Lominska CE, Badkul RK, Jiang H, Wang F. Volumetric Modulated Arc Therapy (VMAT) Significantly Reduces Treatment Time and Monitor Units for Whole-Brain Radiation Therapy (WBRT) While Still Meeting RTOG Protocol 0933. Hippocampal Sparing Constraints. 2015; 93(3):E621.

31. Rong Y, Evans J, Xu-Welliver M, Pickett C, Jia G, Chen Q, Zuo L. Dosimetric evaluation of intensity-modulated radiotherapy, volumetric modulated arc therapy, and helical tomotherapy for hippocampal-avoidance whole brain radiotherapy. PLoS One. 2015;10(4):e0126222.

32. Jiang A, Sun W, Zhao F, Wu Z, Shang D, Yu Q, Wang S, Zhu J, Yang F, Yuan $S$. Dosimetric evaluation of four whole brain radiation therapy approaches with hippocampus and inner ear avoidance and simultaneous integrated boost for limited brain metastases. Radiat Oncol. 2019;14(1):46.

33. Kim KS, Seo SJ, Lee J, Seok JY, Hong JW, Chung JB, Kim E, Choi N, Eom KY, Kim JS, Kim IA. Inclined head position improves dose distribution during hippocampal-sparing whole brain radiotherapy using VMAT. Strahlenther Onkol. 2016;192(7):473-80.

34. Moon SY, Yoon M, Chung M, Chung WK, Kim DW. Comparison of the extent of hippocampal sparing according to the tilt of a patient's head during WBRT using linear accelerator-based IMRT and VMAT. Phys Med. 2016;32(5):657-63

35. Zheng J, Aljabab S, Lacasse P, Bahm J, Lekx-Toniolo K, Grimard L. Functional cranio-spinal irradiation: A hippocampal and hypothalamic-pituitary axis sparing radiation technique using two IMRT modalities. Med Dosim. 2020; 45(2):190-6. 
36. Gondi V, Tome WA, Marsh J, Struck A, Ghia A, Turian JV, Bentzen SM, Kuo JS, Khuntia D, Mehta MP. Estimated risk of perihippocampal disease progression after hippocampal avoidance during whole-brain radiotherapy: safety profile for RTOG 0933. Radiother Oncol. 2010;95(3):327-31.

37. Janssen S, Mehta P, Bartscht T, Schmid SM, Fahlbusch FB, Rades D.

Prevalence of metastases within the hypothalamic-pituitary area in patients with brain metastases. Radiat Oncol. 2019;14(1):152.

\section{Publisher's Note}

Springer Nature remains neutral with regard to jurisdictional claims in published maps and institutional affiliations.

Ready to submit your research? Choose BMC and benefit from:

- fast, convenient online submission

- thorough peer review by experienced researchers in your field

- rapid publication on acceptance

- support for research data, including large and complex data types

- gold Open Access which fosters wider collaboration and increased citations

- maximum visibility for your research: over $100 \mathrm{M}$ website views per year

At BMC, research is always in progress.

Learn more biomedcentral.com/submissions 